\title{
Factors Influencing Warfarin Requirements -Warfarin Increases Lactate Dehydrogenase Concentration in Patients with Valve Prostheses-
}

\author{
Kazuo Sakaguchi*1, Keiko lizuka ${ }^{2}$, Ryuya Horiuchi ${ }^{2}, K_{\text {Koko Takahashi }}{ }^{3}$, Haruzo Satoh ${ }^{4}$, \\ Atsushi Kobayashi ${ }^{5}$ and Shuichi Ichikawa ${ }^{6}$ \\ Department of Pharmacy ${ }^{1}$ and Internal Medicine ${ }^{6}$, Cardiovascular Hospital of Central Japan \\ Department of Pharmacy, Gunma University Hospital ${ }^{2}$ \\ Department of Pharmacy, Public Tomioka General Hospital ${ }^{3}$ \\ Department of Pharmacy, Isesaki Municipal Hospital ${ }^{4}$ \\ Department of Pharmacy, Maebashi Red Cross Hospital ${ }^{5}$
}

$\left[\begin{array}{l}\text { Received November 10, } 2003 \\ \text { Accepted July 8, } 2004\end{array}\right]$

We carried out a retrospective medical chart survey to clarify the effects of various patient factors on warfarin dose requirements. Age, body weight, biochemical data and daily warfarin dose were recorded for 332 outpatients stabilized on warfarin. We found that there was a negative correlation between age and therapeutic warfarin dose $(\mathrm{r}=-0.3089, \mathrm{p}<0.01$, $\mathrm{n}=332)$, and positive correlations between body weight and therapeutic warfarin dose $(\mathrm{r}=0.1623, \mathrm{p}<0.05, \mathrm{n}=213)$ and lactate dehydrogenase $(\mathrm{LDH})$ concentration and therapeutic warfarin dose $(\mathrm{r}=0.2071, \mathrm{p}<0.01, \mathrm{n}=328)$.

The mean serum LDH levels in patients with and without valve prostheses were $561.82 \pm 151.06$ and $393.61 \pm 113.66$ $\mathrm{IU} / \mathrm{L}$, respectively $(\mathrm{p}<0.01)$, suggesting the release of $\mathrm{LDH}$ due to red cell hemolysis in patients with valve prostheses. Moreover, the correlation coefficient between LDH concentration and therapeutic warfarin dose in patients with valve prostheses was higher than that in patients without valve prostheses, such greater correlation between LDH concentration and warfarin dose also indicating warfarin-induced release of LDH from red blood cells in patients with valve prostheses.

Key words — warfarin, lactate dehydrogenase, anticoagulants, valve replacement, medical chart survey

\section{Introduction}

Oral anticoagulant therapy with warfarin is essential for optimal management of many thromboembolic and vascular disorders. Since warfarin has a very narrow therapeutic range, close monitoring of anticoagulant therapy is required to avoid significant bleeding.

It has been reported that elderly patients receiving oral anticoagulant therapy have a greater risk for bleeding than younger patients ${ }^{1)}$. Of the various factors that influence an individual's response to the warfarin, the relationship between age and sensitivity to warfarin has been investigated extensively ${ }^{2-5)}$. These studies have revealed that the sensitivity to oral anticoagulants increases with age. Therefore, age is a important predictor of therapeutic warfarin dose.

Warfarin is highly bound to plasma albumin ${ }^{6}$, having 1 to 3 primary binding sites per molecule of albumin in humans at a physiologic $\mathrm{pH}$. Therefore, it is important to carefully monitor the anticoagulant response to warfarin in hypoalbuminemic patients.

It has been reported that R-warfarin was more cytotoxic in rat hepatocyte primary cultures than other drugs, including phenylbutazone, furosemide, acetaminophen, sodium salicylate, sodium chloride, based on measuring lactate dehydrogenase $(\mathrm{LDH})$ released into the culture medium ${ }^{7}$. If warfarin exhibits a similar cytotoxicity in human hepatocytes, intracellular LDH may be released, and there would be a significant relationship between warfarin dose and LDH concentration.

To investigate the factors that may be determinants of the response to a given warfarin dose in an individual patient, we performed a retrospective medical chart survey, and assessed both the effect of various factors on the anticoagulant response to warfarin and the effect of warfarin dose on the release of $\mathrm{LDH}$. 


\section{Methods}

\section{Patients}

The records of 332 outpatients stabilized on warfarin for prophylaxis of thromboembolic disease at the department of cardiology or cardiovascular surgery of Cardiovascular Hospital of Central Japan, Gunma University Hospital, Gunma Prefectural Cardiovascular Center, Public Tomioka General Hospital, Isesaki Municipal Hospital, and Maebashi Red Cross Hospital were retrospectively reviewed. Only those patients whose warfarin dose had achieved steady state were included. Patients who were suspected of noncompliance were excluded from this study.

\section{Medical Survey}

The means of the values of the thrombo tests and prothrombin times were $27.9 \pm 17.3 \%(n=325)$ and $17.1 \pm$ $5.1 \mathrm{sec}(\mathrm{n}=298)$, respectively. Age, body weight, thrombo test, prothrombin time, total protein concentration (TP), albumin concentration (ALB), albumin/globulin $(\mathrm{A} / \mathrm{G})$ ratio, glutamate oxaloacetate transaminase activity (GOT), glutamate pyruvate transaminase activity (GPT), alkaline phosphatase activity (ALP), lactate dehydrogenase activity (LDH), serum creatinine ( $\mathrm{SCr}$ ), and daily warfarin dose were recorded inspecting medical charts between January and October in 1995.

\section{Statistical Analysis}

Values are expressed as the means \pm standard deviation (S. D.). Correlation coefficients ( $r$ ) and the regression equation are reported along with the $\mathrm{p}$ value. Comparison of mean values was made by Student's t-test for unpaired data. $\mathrm{P}$ values less than 0.05 were considered statistically significant.

\section{Results}

\section{Effects of Various Factors of Patients on Warfarin Doses}

Fig. 1 shows the distribution of daily warfarin doses in 332 outpatients on a stable, therapeutic warfarin dose. The mean dose was $3.09 \pm 1.20 \mathrm{mg} / \mathrm{day}$, but the doses ranged widely, from 1 to $9 \mathrm{mg} /$ day. The majority of the therapeutic daily warfarin doses was between 2 and $6 \mathrm{mg}(89.2 \%)$.

Table 1 shows the relationship between daily outpatient warfarin dose and various factors. There was a negative correlation between age and therapeutic warfarin dose $(\mathrm{r}=-$ $0.3089, \mathrm{p}<0.01, \mathrm{n}=332$ ) and a weak, but statistically significant positive correlation between body weight and therapeutic warfarin dose $(\mathrm{r}=0.1623, \mathrm{p}<0.05, \mathrm{n}=213)$ and $\mathrm{A} / \mathrm{G}$ ratio and warfarin dose $(r=0.1111, p<0.05, n=328)$. There was no correlation between therapeutic warfarin dose and the other factors.

\section{Relationship between LDH Concentration and Warfarin} Dose in Patients with and without Valve Prostheses

The correlation between LDH concentration and therapeu-

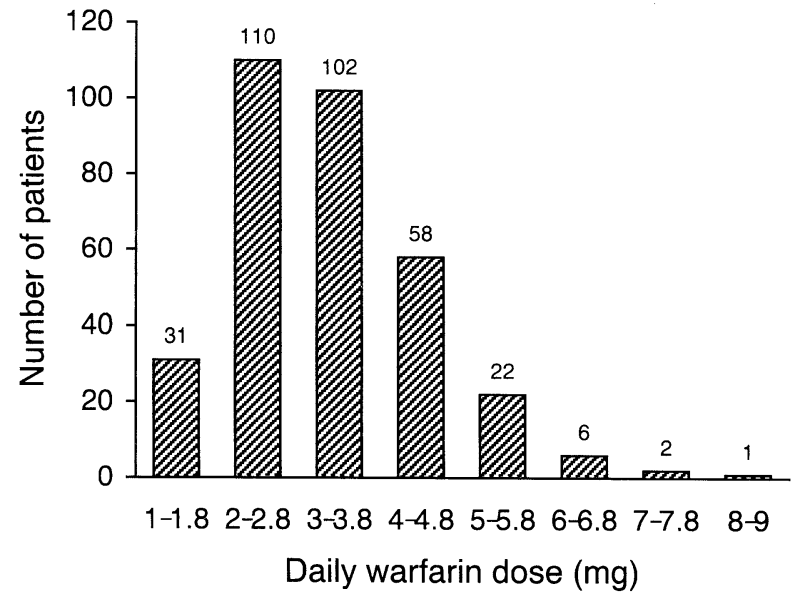

Fig. 1. Distribution of Daily Warfarin Doses.

tic warfarin dose is shown in Fig. 2. There was a significant positive correlation between LDH concentration and therapeutic warfarin dose $(r=0.2071, p<0.01, n=328)$.

If $\mathrm{LDH}$ concentration is elevated after valve replacement by hemolysis, serum LDH concentrations reflect the rate of red cell breakdown by implantation of prosthetic material into the heart. Therefore, we evaluated the relationship between LDH concentration and warfarin dose in patients with and without valve prostheses, to elucidate the participation of valve replacement in elevation of LDH concentration by warfarin (Fig. 3). There was a positive correlation between LDH concentration and warfarin dose in patients with valve prostheses $(r=0.1608, p=0.0524, n=146)$ (Fig. $3 \mathrm{~A})$, and no correlation in patients without valve prostheses $(\mathrm{r}=$ $0.0542, \mathrm{p}=0.5107, \mathrm{n}=150$ ) (Fig. $3 \mathrm{~B}$ ). However, there was statistically no relationship in the correlation coefficient between patients with and without valve prostheses.

Table 2 shows LDH concentrations and therapeutic warfarin doses of patients with or without valve prostheses. The mean serum LDH activities of patients with and without valve prostheses were $561.82 \pm 151.06$ and $393.61 \pm 113.66$ $\mathrm{IU} / \mathrm{L}$, respectively $(\mathrm{p}<0.01)$, and the mean therapeutic warfarin doses of patients with and without valve prostheses were $3.40 \pm 1.20$ and $2.92 \pm 1.17 \mathrm{mg}$, respectively $(\mathrm{p}<0.01$ ). The mean thrombo tests of patients with and without valve prostheses were $25.8 \pm 13.0 \%(n=144)$ and $30.0 \pm 20.7 \%$ $(n=146)$, respectively $(p<0.05)$. It seems reasonable to assume that the lower thrombo test of patients with valve prostheses reflects the significant difference in warfarin doses of patients with and without valve prostheses. On the other hand, there was no elevation of serum LDH concentration in venous thromboembolic disease and coronary artery disease etc. except heart valve replacement.

\section{Discussion}

Many studies concerning the age-related anticoagulant effects of warfarin therapy have been reported ${ }^{2-5)}$. Sensitivity to 
Table 1. Relationship between Daily Warfarin Dose and Patient Factors.

\begin{tabular}{ccccc}
\hline Factor & $\mathrm{n}$ & Mean \pm S.D. & $\mathrm{r}$ & $\mathrm{p}$ \\
\hline Age (years) & 332 & $61.14 \pm 12.76$ & -0.3089 & $<0.01$ \\
Body weight $(\mathrm{kg})$ & 213 & $54.83 \pm 10.69$ & 0.1623 & $<0.05$ \\
TP (g/dL) & 331 & $7.08 \pm 0.56$ & 0.0308 & N.S. \\
ALB (g/dL) & 332 & $4.19 \pm 0.38$ & 0.0923 & N.S. \\
A/G ratio & 328 & $1.49 \pm 0.27$ & 0.1111 & $<0.05$ \\
GOT (IU/L) & 331 & $26.98 \pm 16.50$ & 0.0341 & N.S. \\
GPT (IU/L) & 331 & $21.63 \pm 19.04$ & 0.0246 & N.S. \\
ALP (IU/L) & 269 & $163.54 \pm 63.79$ & -0.0647 & N.S. \\
SCr (mg/dL) & 319 & $0.95 \pm 0.29$ & -0.0428 & N.S. \\
\hline Abbriations
\end{tabular}

Abbreviations used are: TP, total protein; ALB, albumin; A/G, albumin/globulin; GOT, glutamate oxaloacetate transaminase; GPT, glutamate pyruvate transaminase; ALP, alkaline phosphatase; $\mathrm{SCr}$, serum creatinine; N.S., not significant.

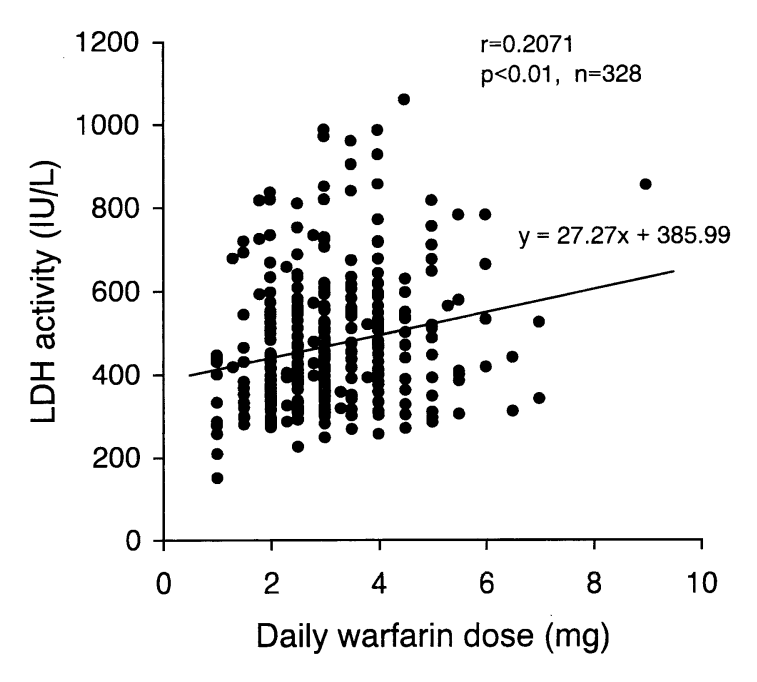

Fig. 2. Relationship between Warfarin Dose and LDH Concentration.

an oral anticoagulant tended to increase with advancing years ${ }^{2}$. Our data also shows that increasing age is associated with an increased sensitivity to warfarin therapy. Since elimination of warfarin by hepatic metabolism is unaltered in the elderly ${ }^{8,9)}$, aging seems to produce a pharmacodynamic change which alters the intrinsic sensitivity to warfarin. Pharmacodynamic factors of patients may be more important than pharmacokinetic factors in the age-related variation of therapeutic warfarin dose.

There was a positive correlation between body weight and warfarin dose in agreement with a previous report ${ }^{10)}$. On the other hand, we found no significant correlation between warfarin dose and albumin levels. It has been reported that an increase in the free fraction of warfarin in the plasma due to hypoalbuminemia may result in increased clearance of warfari $^{11}$. Therefore, differences in serum albumin may be not responsible for the differences in the anticoagulant response to warfarin. Even if a reduction in serum albumin increases the free fraction of warfarin, it seems that this dose not produce a clinically important change in the anticoagulant response to warfarin. However, as there was a positive correlation between $\mathrm{A} / \mathrm{G}$ ratio and warfarin dose, it should be noted that albumin level may influence warfarin dose.

There was no significant relationship between warfarin dose and serum creatinine. Since warfarin is almost exclusively cleared by hepatic metabolism, impaired renal function is unlikely to alter its pharmacokinetics. Therefore, it seems logical that a reduction in renal function has no effect on warfarin sensitivity.

There was no significant correlation between warfarin dose and serum hepatic enzyme concentrations; GOT, GPT, and ALP. On the other hand, there was a positive correlation between warfarin dose and LDH concentration. However, it seems that there is no relationship between serum LDH concentration and anticoagulant response.

It is well known that LDH concentration may be elevated after valve replacement by hemolysis, resulting in a hemolytic anemia ${ }^{12-14)}$. We also showed that serum LDH concentration is elevated after heart valve replacement in patients receiving treatment with warfarin. Therefore, we evaluated both the relationship between LDH concentration and therapeutic warfarin dose of patients with valve prostheses and that of patients without valve prostheses to clarify whether an elevation in LDH is due to leakage from red cells. The correlation coefficient between LDH concentration and therapeutic warfarin dose in patients with valve prostheses was higher than that in patients without valve prostheses 

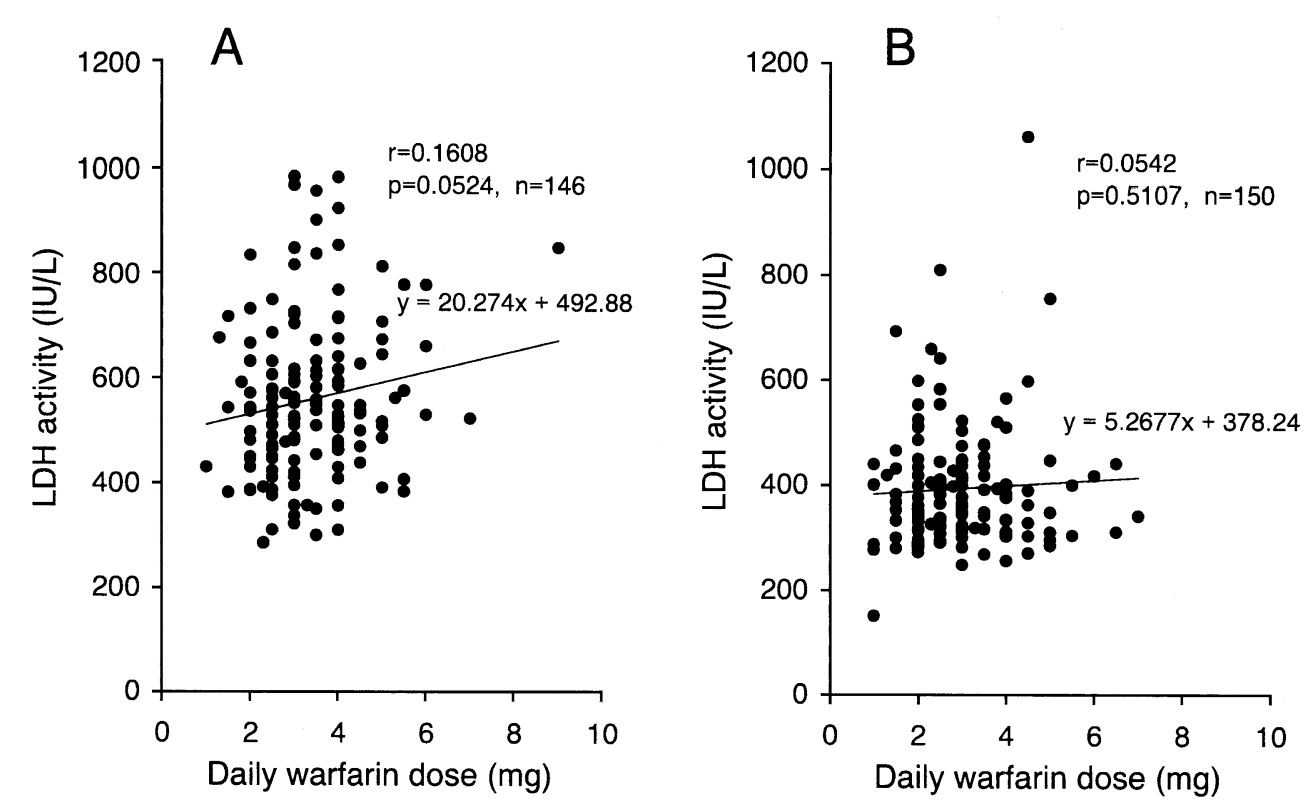

Fig. 3. Relationship between Warfarin Dose and LDH Concentration.

A : in patients with valve prostheses

$\mathrm{B}:$ in patients without valve prostheses

Table 2. LDH Concentrations and Therapeutic Warfarin Doses of Patients with or without Valve Prostheses.

\begin{tabular}{lccc}
\hline & $\begin{array}{c}\text { Patients with valve } \\
\text { prostheses }(\mathrm{n}=146)\end{array}$ & $\begin{array}{c}\text { Patients without valve } \\
\text { prostheses }(\mathrm{n}=150)\end{array}$ & $\mathrm{p}$ \\
\hline LDH (IU/L) & $561.82 \pm 151.06$ & $393.61 \pm 113.66$ & $<0.01$ \\
Warfarin dose $(\mathrm{mg})$ & $3.40 \pm 1.20$ & $2.92 \pm 1.17$ & $<0.01$ \\
\hline
\end{tabular}

(Fig. 3). These results suggest that the relationship between LDH concentration and therapeutic warfarin dose might reflect the rate of red cell breakdown by warfarin.

It has been reported that $\mathrm{LDH}$ concentration is a reliable index for cytotoxicity in rat hepatocyte primary culturs ${ }^{7)}$. The relative cytotoxicities of the studied drugs are:Rwarfarin $>$ phenylbutazone $>$ furosemide $>$ acetoaminophen $>$ sodium salicylate $>$ sodium chloride. The cytotoxic ranking of these six drugs closely resembles their relative cytotoxicity based on a cell proliferation assay using a human embryonic lung cell line, BCL-D $1^{15}$. However, in our study, the therapeutic dose of warfarin did not correlate with serum hepatic enzyme concentrations of GOT and GPT, suggesting that LDH might be released from cells other than hepatocytes.

In conclusion, our study suggests that warfarin cause human red cell breakdown, elevating serum LDH concentration, and that hemolysis is facilitated by warfarin therapy in patients with valve prostheses. Therefore, warfarin therapy of patients with valve prostheses should be meticulously carried out, observing LDH concentration.

It has not been reported that warfarin therapy induces intravascular hemolysis. Although the LDH isozyme should be analyzed in our study to identify the origin of increased $\mathrm{LDH}$, it was impossible to analyze the $\mathrm{LDH}$ isozyme because of a retrospective medical chart survey. It also seems effective that $\mathrm{LDH}$ concentration of patients with valve prostheses is measured before and after the administration of warfarin. Further investigation should be undertaken to elucidate the effect of warfarin on hemolysis.

\section{Acknowledgments}

We thank pharmacists of Gunma Prefectural Cardiovascular Center for their retrospective medical chart survey.

\section{References}

1) S. Husted, F. Andreasen, The influence of age on the response to anticoagulants, Br. J. Clin. Pharmacol., 4, 559-565 (1977).

2) M. Toohey, Clinical trial of phenylindanedione as an anticoagulant, Br. Med. J., 21, 650-652 (1953).

3) J.H. Gurwitz, J. Avorn, D. Ross-Degnan, I. Choodnovskiy, J. Ansell, Aging and the anticoagulant response to warfarin therapy, Ann. Intern. Med., 116, 901-904 (1992). 
4) M. Redwood, C. Taylor, B.J. Bain, J.H. Matthews, The association of age with dosage requirement for warfarin, Age Ageing, 20, 217-220 (1991).

5) H. Wynne, L. Cope, P. Kelly, T. Whittingham, C. Edwards, F. Kamali, The influence of age, liver size and enantiomer concentrations on warfarin requirements, $\mathrm{Br}$. J. Clin. Pharmacol ., 40, 203-207 (1995).

6) R.A. O'Reilly, The binding of sodium warfarin to plasma albumin and its displacement by phenylbutazone, Ann. N.Y. Acad. Sci., 226, 293-308 (1973).

7) E.S. Chao, D. Dunbar, L.S. Kaminsky, Intracellular lactate dehydrogenase concentration as an index of cytotoxicity in rat hepatocyte primary culture, Cell Biol. Toxicol., 4, 1-11 (1988).

8) P.A. Routledge, D.H. Chapman, D.M. Davies, M.D. Rawlins, Pharmacokinetics and pharmacodynamics of warfarin at steady state, Br. J. Clin. Pharmacol., 8, 243-247 (1979).

9) A.M.M. Shepherd, D.S. Hewick, T.A. Moreland, I.H. Stevenson, Age as a determinant of sensitivity to warfarin, Br. J. Clin. Pharmacol., 4, 315-320 (1977).
10) P.A. Routledge, P.H. Chapman, D.M. Davies, M.D. Rawlins, Factors affecting warfarin requirements, Europ. J. Clin. Pharmacol., 15, 319-322 (1979).

11) R.J. Piroli, G.T. Passananti, C.A. Shively, E.S. Vesell, Antipyrine and warfarin disposition in a patient with idiopathic hypoalbuminemia, Clin. Pharmacol. Ther., 30, 810-816 (1981).

12) D.W. Stein, S.H. Rahimtoola, F.E. Kloster, R. Selden, A. Starr, Thrombotic phenomena with nonanticoagulated, composite-strut aortic prostheses, J. Thorac. Cardiovasc. Surg., 71, 680-684 (1976).

13) J. Dale, E. Myhre, Intravascular hemolysis in the late course of aortic valve replacement. Relation to valve type, size, and function, Am. Heart J., 96, 24-30 (1978).

14) R.M. Saad, M.W. Wolfe, Progressive hemolytic anemia due to delayed recognition of a Beall mitral valve prosthesis, Chest, 99, 496-498 (1991).

15) P. Knox, P.F. Uphill, J.R. Fry, J. Benford, M. Balls, The FRAME MULTICENTRE project on in vitro cytoxicology, Food Chem. Toxicol., 24, 457-463 (1986). 